\title{
Sequential Intrahepatic Metabolic Effects of Enteric Galactose Alimentation in Newborn Rats
}

\author{
R. M. KLIEGMAN AND S. MORTON \\ Department of Pediatrics, Case Western Reserve University, Rainbow Babies and Children's Hospital, \\ Cleveland, Ohio 44106
}

\begin{abstract}
We determined metabolic responses after enteric galactose alimentation in 5- to 7-day-old newborn rats fasted for $24 \mathrm{~h}$. The glycemic response was attenuated after enteric galactose feeding compared with the response after enteric glucose-fed rat pups. ${ }^{14} \mathrm{C}$ radioactivity in blood from galactose-fed pups was reduced as counts in blood galactose were lower than counts in blood glucose in glucose-fed pups. Nonetheless within $15 \mathrm{~min},\left[{ }^{14} \mathrm{C}\right]$ from galactose appeared in blood glucose suggesting rapid conversion of galactose to glucose. The plasma insulin response was also attenuated after galactose feeding compared with the insulin response after enteric glucose. Hepatic glycogen content increased rapidly after enteric galactose feeding and was higher than after glucose feeding at 60,120 , and $180 \mathrm{~min}$. Significant glycogen synthesis after oral glucose was delayed and occurred at $240 \mathrm{~min}$. Carbon radioactivity in glycogen was higher in galactose fed pups between 15 and $360 \mathrm{~min}$ of the study. Serial determination of hepatic metabolites revealed an increase of galactose-1-phosphate levels after oral galactose at $\mathbf{2 4 0}$ and $300 \mathrm{~min}$ and a transient decline of ATP at $15 \mathrm{~min}$. Other hepatic metabolites did not demonstrate significant differences between the two groups. These data suggest that hepatic glycogen synthesis is more rapid and occurs sooner after galactose than after glucose alimentation in previously fasted newborn rats. Galactose may enter a more direct pathway for neonatal hepatic glycogen synthesis. The relatively delayed entry of glucose label into hepatic glycogen and the delay of net glycogen synthesis after oral glucose suggest that glucose entry is not direct and may require further metabolism before incorporation into glycogen. Although galactose may replenish fasting neonatal hepatic glycogen content faster than glucose, there is concern over the elevated hepatic galactose-1-phosphate levels and the transient decline of ATP. (Pediatr Res 24: 302307, 1988)
\end{abstract}

\section{Abbreviations}

UDP-glucose, uridine diphosphate glucose

PCA, perchloric acid

Galactose is an important hexose source during the neonatal period. Galactose represents $50 \%$ of calories derived from carbohydrates among newborn mammals fed lactose-containing milk (1). In addition to serving as a substrate for oxidative metabolism and gluconeogenesis, galactose may also be a major

Received February 1, 1988; accepted April 25, 1988.

Correspondence Robert M. Kliegman, M.D., Department of Pediatrics, Rainbow Babies and Children's Hospital, 2101 Adelbert Road, Cleveland, OH 44106.

Supported by a grant from the Rainbow Board of Trustees and Ross Laborato- precursor for neonatal hepatic glycogen synthesis (2-6). Previous investigations from our laboratory and other laboratories have demonstrated enhanced incorporation of galactose into glycogen when compared with glucose $(2,3,6)$. Additional investigations have demonstrated that hepatic galactose uptake exceeds that for glucose in vitro, whereas activation of hepatic glycogen synthase and inactivation of glycogen phosphorylase was greater in the presence of galactose than that for glucose $(5,6)$. These data suggest that galactose or a metabolite of galactose may regulate the enzymes of hepatic glycogen synthesis. Alternately, the enhanced incorporation of galactose into glycogen relative to glucose may result from differences of the metabolic pathways of these two hexoses (3).

Our previous investigations of the metabolic differences between galactose and glucose studied glucose kinetics in addition to the metabolic pertubations in neonatal canine liver (7). Hepatic metabolic alterations include an equivalent increase in glycogen content after the administration of glucose and galactose. However, radiolabeled carbon incorporation into glycogen from ${ }^{14} \mathrm{C}$ galactose was 10 -fold more than that after ${ }^{14} \mathrm{C}$ glucose (2). In these investigations hepatic tissue sampling was delayed and occurred after completion of the kinetic studies. This delay was 3-4 $\mathrm{h}$ after the carbohydrate load (7). Blood levels of galactose were maximum 30 min after ingestion and returned to very low baseline levels within $90 \mathrm{~min}$. Because hepatic tissue was sampled 2-4 h after the peak of blood galactose levels, the immediate effects of hexose alimentation on hepatic glycogen synthesis may not be apparent. Delaying the sampling of tissue by this period of time may mask the perturbations of hepatic metabolism by galactose. Presently there is a paucity of investigations on the immediate effects of galactose on neonatal hepatic metabolism. The present study investigates the effects of enteric galactose alimentation on pertubations of hepatic metabolites from 15 to $360 \mathrm{~min}$ after hexose administration. The results suggest that galactose is rapidly incorporated into hepatic glycogen despite lower plasma insulin levels. Furthermore, glycogen synthesis from glucose was delayed and appeared to occur from an indirect pathway.

\section{MATERIALS AND METHODS}

Materials. D-[U- ${ }^{14}$ C]glucose (SA $144 \mu \mathrm{Ci} / \mathrm{mmol}$ ), and D-[U${ }^{14} \mathrm{C}$ galactose (SA $144 \mu \mathrm{Ci} / \mathrm{mmol}$ ) were purchased from New England Nuclear (Boston, MA). According to New England Nuclear specifics on this lot, the galactose was contaminated with less than $0.1 \%$ glucose. After derivatization and chromatographic separation the actual contamination was equivalent to background counts. All enzymes were purchased from Sigma Chemical Co. (St. Louis, MO). Scintisol was purchased from Isolab Inc. (Akron, OH). Galactose (Sigma) and glucose (Mallinckrodt, St. Louis, MO) contained no detectable contamination with glucose or galactose, respectively. All chemicals and standards were analytical and reagent grade. 
Animals. This study was approved by the Case Western Reserve University Animal Welfare Committee. Pregnant Sprague Dawley rats with known dates of confinement were housed in cages and had free access to food and water in a light-dark cycled environment. Pregnant rats delivered spontaneously and the pups remained with the mothers in her cage for 4-6 days for nurturing, warmth, and general care. On day 4-6 the pups were removed from the mother's cage and placed in a warmed humidified incubator where they were fasted for $24 \mathrm{~h}$.

Experimental design. Pups were weighed (7-12 g) and received an enteric feeding of $0.7 \mathrm{mg} / \mathrm{g}$ of either [ $\mathrm{U}-{ }^{14} \mathrm{C}$ ]galactose, 0.25 $\mu \mathrm{Ci} / \mathrm{g}$ or $\left[\mathrm{U}-{ }^{14} \mathrm{C}\right]$ glucose $0.25 \mu \mathrm{Ci} / \mathrm{g}$. The volume of this feed was $0.05 \mathrm{ml} / \mathrm{g}$ and was delivered by PE-50 tubing attached to a $1-\mathrm{ml}$ spring. The tubing was placed carefully into the stomach of each rat without signs of gasping, cyanosis, or alterations of behavior after the feeding. Control or zero time rats were fed normal saline.

Pups were killed 15, 30, 60, 120, 180, 240, 300, and $360 \mathrm{~min}$ after enteric carbohydrate alimentation. Sample size varied from four to nine pups per each time point for each determination. Blood was sampled from the carotid vessels after decapitation whereas hepatic tissue was simultaneously and quickly (less than $5 \mathrm{~s}$ ) freeze clamped to the temperature of liquid nitrogen between aluminum blocks. Blood and liver were then stored at $-80^{\circ} \mathrm{C}$.

Analyses. Circulating substrates. Blood was precipitated with $10 \%$ perchloric acid (PCA) and then neutralized with $\mathrm{K}_{2} \mathrm{CO}_{3}$. Glucose was assayed as reported before (8). Galactose was fluorometrically determined using galactose dehydrogenase (9). Plasma insulin was assayed by double antibody radioimmunoassay (Amersham, Arlington Heights, IL). All analyses were run in duplicate and adapted to micromethods.

Hepatic determinations. Hepatic glycogen, $\left[{ }^{14} \mathrm{C}\right]$ label incorporation into glycogen and $\left[{ }^{14} \mathrm{C}\right]$ glucose-specific activity in glycogen were determined after homogenization of liver and subsequent alcohol precipitation at $0^{\circ} \mathrm{C}$. Hepatic glycogen was further purified after two ether washes. Glycogen was then redissolved in distilled water and assayed enzymatically according to Vannucci and Duffy (10). An aliquot of glycogen was then simultaneously counted on a $\beta$-scintillation counter to determined the $\left[{ }^{14} \mathrm{C}\right]$ content of the glycogen.

A second sample of hepatic tissue was pulverized to a fine powder at the temperature of dry ice, extracted with $3 \mathrm{M}$ PCA and 10 mM EDTA, and then neutralized with $\mathrm{K}_{2} \mathrm{CO}_{3}$. Standard fluorometric assays were used to determine hepatic, UDP-glucose, galactose-1-phosphate, fructose-6-phosphate, fructose 1, 6 diphosphate, phosphoenolpyruvate, pyruvate, lactate, citrate, and ATP $(7,9)$. Galactose-1-phosphate was determined because elevated levels are associated with toxicity in galactosemia.

An additional sample of liver was homogenized at $0^{\circ} \mathrm{C}$ and assayed to determine glycogen synthase and phosphorylase activities according to Schwartz and Rall (11).

Glucose radioactivity. Neutralized samples of PCA-treated blood were evaporated to dryness and were then reconstituted with unlabeled water. Before placement of this neutralized sample on a Dowex AG 1-X8 column, galactose was completely converted to galactonate with galactose dehydrogenase (2). After this derivatization step the sample was applied to the column. Glucose was first eluted in the water wash. Thereafter, galactonate was eluted with $0.02 \mathrm{~N} \mathrm{HCl}$. After glucose and the derivatized galactose were eluted, lactate and additional three carbon substrates were removed with $0.05 \mathrm{~N} \mathrm{HCl}$. This method completely separated glucose from galactose and three carbon substrates and resulted in more than $95 \%$ recovery of labeled compounds.

Radioactivity in ${ }^{14} \mathrm{C}$ was counted by liquid scintillation for 10 min using external and internal standards. All counts were corrected for background, blanks, and quenching and were expressed as disintegrations/min.

Statistical analyses. Tabular notations are recorded as the mean \pm SEM. Statistical analyses were used according to stand- ard computer programs for the Student's $t$ test. Comparisons are made predominantly between time-matched pups fed glucose or galactose and significance is reported in the tables or figures. Longitudinal changes across time are reported in the text and were analyzed with analyses of variance. On initial evaluation of the data we realized that most of the effects occurred sooner than $360 \mathrm{~min}$, thus we concentrated the remaining experiments at earlier time points. When sufficient pups were studied at 360 min, we reported that data.

\section{RESULTS}

Circulating glucose, galactose, insulin, and radioactivity. Blood glucose increased markedly after enteric glucose alimentation and achieved peak values within $60 \mathrm{~min}$ of feeding (Fig. 1). The glycemic response after an equivalent galactose feeding was markedly reduced as blood glucose levels increased but were significantly lower than blood glucose levels after enteric glucose administration at 15,30 , and 60 min of study. In both groups, blood glucose returned to fasting basal levels $300 \mathrm{~min}$ after enteric carbohydrate alimentation.

After galactose alimentation blood galactose levels increased from concentrations less than 0.1 to $3.7 \mathrm{mM}$ within $15 \mathrm{~min}$ (Fig. 1). After this time blood galactose levels declined rapidly and achieved values comparable to glucose fed pups within $180 \mathrm{~min}$ of the study. There was no increment of blood galactose concentrations in glucose-fed pups.

Plasma insulin concentrations paralleled the glycemic responses noted in Figure 1 in both pup groups (Fig. 2). Plasma insulin levels achieved peak values between 30 and $60 \mathrm{~min}$. Insulin concentrations were more after enteric glucose feeding than those for galactose-fed pups at $60 \mathrm{~min}$. Insulin levels returned to basal fasting concentrations by $180 \mathrm{~min}$ of the study.

Within 15 min of ${ }^{14} \mathrm{C}$ glucose feeding radioactivity appeared in the blood (Fig. 3). The amount of radioactivity in glucose increased and achieved peak values at $60 \mathrm{~min}$ of the study. The curve of radioactivity was similar to that for the actual glycemic response after glucose feeding as recorded in Figure 1. Radioactivity appearing in blood galactose was also rapid and was equivalent to the total counts observed after glucose feeding at $15 \mathrm{~min}$ (Fig. 3). Thereafter, counts in blood galactose declined slowly. The curve for appearance of ${ }^{14} \mathrm{C}$ labeled galactose paralleled the curve for assayable galactose as noted in Figure $1 .{ }^{14} \mathrm{C}$ label appearing in blood glucose after ${ }^{14} \mathrm{C}$ galactose feeding was less than that in blood ${ }^{14} \mathrm{C}$ galactose at $15 \mathrm{~min}$ (Fig. 3). After this time point, counts in blood galactose declined whereas the ap-

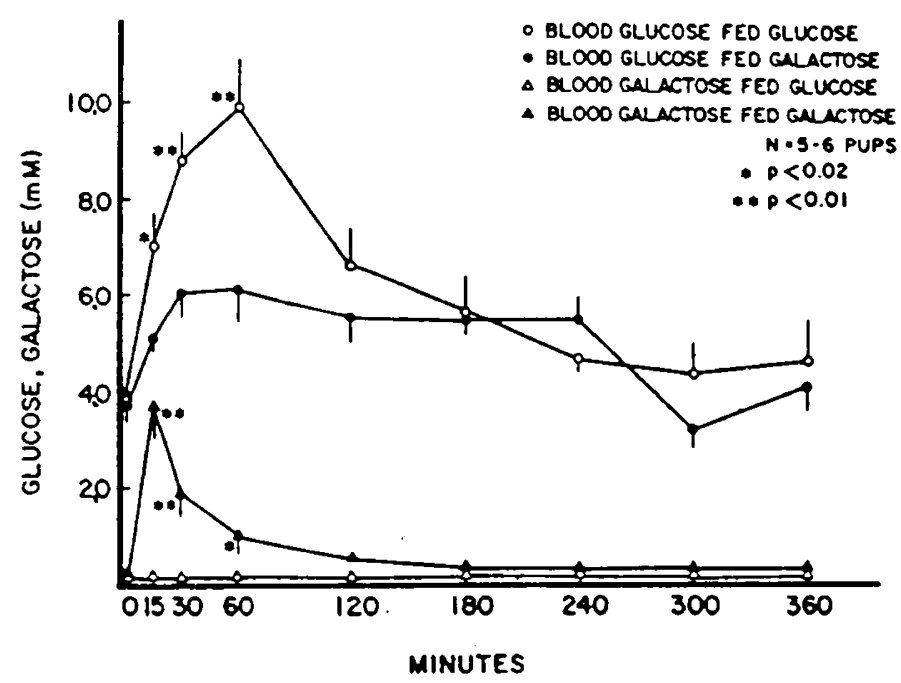

Fig. 1. Blood glucose and galactose concentrations after carbohydrate feeding (y axis). Results are mean \pm SEM. 


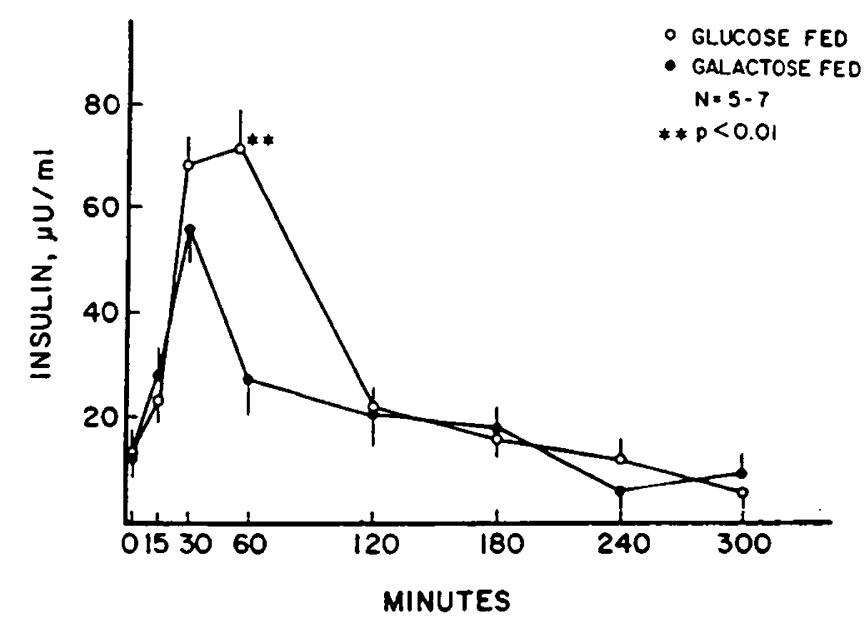

Fig. 2. Plasma insulin response to glucose or galactose alimentation. Results are mean \pm SEM.

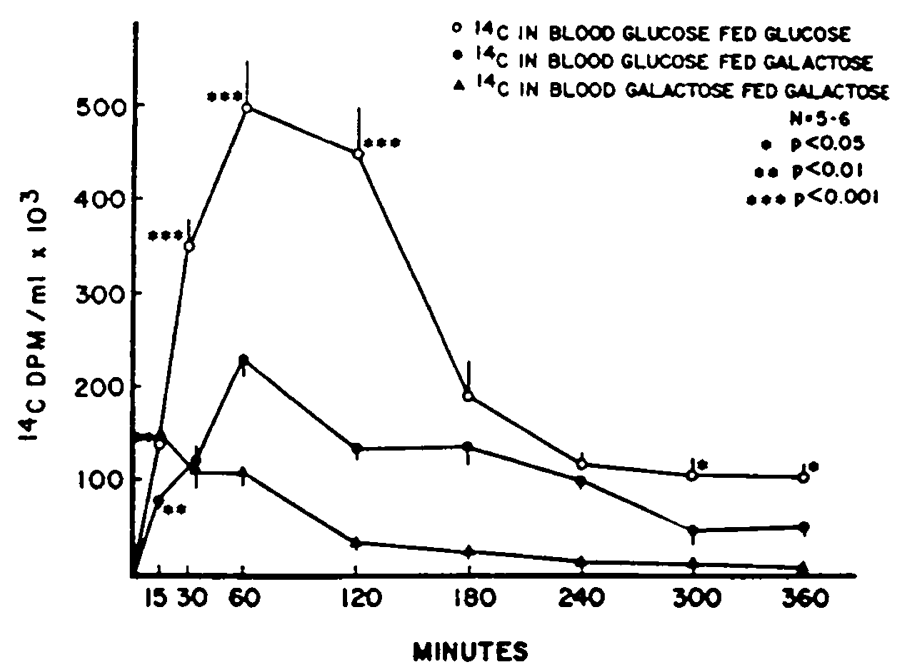

Fig. 3. Radioactivity in blood hexoses after enteric glucose or galactose feeding. Results are mean \pm SEM.

pearance of counts from galactose into blood glucose increased and achieved peak levels at $60 \mathrm{~min}$.

Hepatic glycogen content and radioactivity. Hepatic glycogen levels were equivalent in both groups of pups 15 min after carbohydrate feeding and did not increase above baseline fasting concentrations at this time (Fig. 4). After galactose alimentation hepatic glycogen content increased at $30 \mathrm{~min}$ and achieved peak values at $60 \mathrm{~min}$. Hepatic glycogen content was greater after galactose than after glucose alimentation at 60, 120, and 180 min of the study as glycogen synthesis was delayed in glucosefed rats. After $240 \mathrm{~min}$, glucose-fed pups demonstrated a marked increase of hepatic glycogen levels (Fig. 4). At this time period blood glucose, plasma insulin, and ${ }^{14} \mathrm{C}$-labeled glucose had already declined to fasting levels or levels that were within $20 \%$ of peak ${ }^{14} \mathrm{C}$ counts in blood.

After equivalent quantities of hexose, ${ }^{14} \mathrm{C}$ incorporation into hepatic glycogen from galactose exceeded that for ${ }^{14} \mathrm{C}$ incorporation after glucose at each time point studied (Fig. 5). Galactose label appeared rapidly in glycogen (within $15 \mathrm{~min}$ ) and did not achieve peak values until 120 to $300 \mathrm{~min}$ when an apparent plateau of incorporation into glycogen was noted (Fig. 5). The curve for incorporation of ${ }^{14} \mathrm{C}$ label after galactose feeding paralleled the curve for total assayable glycogen synthesis after galactose alimentation (Fig. 4).

In contrast to the results after galactose feeding, label incorporation into glycogen after glucose alimentation was attenuated,

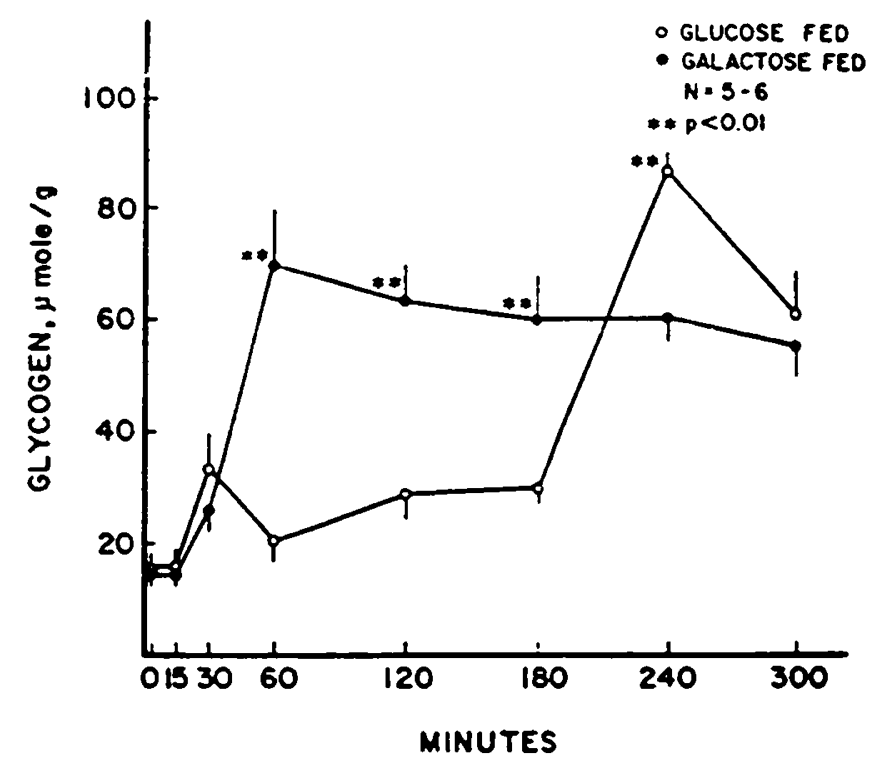

Fig. 4. Hepatic glycogen content in glucose or galactose-fed pups. Results are mean \pm SEM.

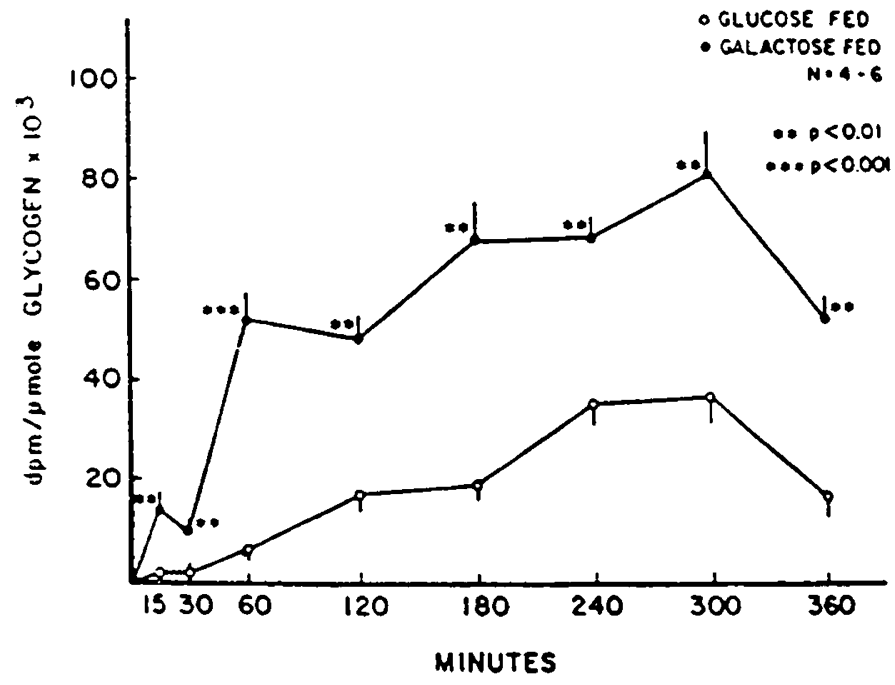

Fig. $5 .{ }^{14} \mathrm{C}$-specific activity in hepatic glycogen after glucose or galactose feeding.

and increased slowly throughout the study period, achieving peak values between $240-300 \mathrm{~min}$. The total counts incorporated into glycogen after glucose alimentation were $50 \%$ of the counts incorporated after galactose alimentation.

Hepatic metabolites and enzymes. To identify potential areas of hepatic regulation after enteric carbohydrate alimentation, key regulatory intermediates of carbohydrate metabolism were determined in newborn rats fed either glucose or galactose. Because galactose-1-phosphate accumulation is associated with potential toxicity in galactosemia, this intermediate was also determined.

After glucose alimentation there were no statistical alterations of serial galactose-1-phosphate levels (Table 1). In contrast, after galactose feeding galactose-1-phosphate levels increased compared with basal levels at $120(p<0.05)$ and $240(p<0.05)$ min (Table 1). Compared with time-matched glucose-fed pups, galactose-fed pups had elevated galactose-1-phosphate concentrations at 30,240 , and 300 min of the study.

Compared with fasted pups, hexose feeding resulted in a decline of hepatic UDP-glucose concentrations at $60 \mathrm{~min}(p<$ $0.05)$ for glucose fed and at 60 and $180 \mathrm{~min}(p<0.05)$ for galactose-fed pups. There were no significant alterations between 
Table 1. Sequential hepatic metabolites in newborn rats*

\begin{tabular}{|c|c|c|c|c|c|c|c|c|}
\hline \multirow{2}{*}{$\begin{array}{c}\text { Oral } \\
\text { substrate }\end{array}$} & \multirow{2}{*}{$\begin{array}{c}\text { Hepatic } \\
\text { metabolite }\end{array}$} & \multicolumn{7}{|c|}{ Time (min) } \\
\hline & & 0 & 15 & 30 & 60 & 120 & 180 & 240 \\
\hline Glucose & Galactose- & $0.146 \pm 0.029$ & $0.117 \pm 0.029$ & $0.154 \pm 0.013$ & $0.185 \pm 0.061$ & $0.196 \pm 0.095$ & $0.140 \pm 0.012$ & $0.139 \pm 0.015$ \\
\hline Galactose & 1-phosphate & $0.146 \pm 0.029$ & $0.140 \pm 0.030$ & $0.246 \pm 0.046 \dagger$ & $0.207 \pm 0.046$ & $0.263 \pm 0.031$ & $0.180 \pm 0.031$ & $0.291 \pm 0.065 \dagger$ \\
\hline Glucose & UDP-glucose & $0.114 \pm 0.017$ & $0.087 \pm 0.015$ & $0.107 \pm 0.024$ & $0.073 \pm 0.007$ & $0.092 \pm 0.009$ & $0.093 \pm 0.018$ & $0.114 \pm 0.014$ \\
\hline Galactose & & $0.114 \pm 0.017$ & $0.104 \pm 0.020$ & $0.096 \pm 0.013$ & $0.064 \pm 0.013$ & $0.101 \pm 0.013$ & $0.069 \pm 0.006$ & $0.102 \pm 0.011$ \\
\hline Glucose & Glucose- & $0.045 \pm 0.008$ & $0.087 \pm 0.015$ & $0.071 \pm 0.029$ & $0.099 \pm 0.020$ & $0.095 \pm 0.014$ & $0.097 \pm 0.030$ & $0.080 \pm 0.013$ \\
\hline Galactose & 6-phosphate & $0.045 \pm 0.008$ & $0.081 \pm 0.014$ & $0.081 \pm 0.017$ & $0.113 \pm 0.039$ & $0.079 \pm 0.014$ & $0.084 \pm 0.010$ & $0.085 \pm 0.010$ \\
\hline Glucose & PEP & $0.106 \pm 0.020$ & $0.089 \pm 0.036$ & $0.110 \pm 0.028$ & $0.194 \pm 0.060$ & $0.150 \pm 0.023$ & $0.199 \pm 0.034$ & $0.156 \pm 0.021$ \\
\hline Galactose & & $0.106 \pm 0.020$ & $0.202 \pm 0.068 \dagger$ & $0.150 \pm 0.026$ & $0.177 \pm 0.048$ & $0.154 \pm 0.021$ & $0.187 \pm 0.024$ & \\
\hline Glucose & Pyruvate & $0.108 \pm 0.018$ & $0.050 \pm 0.009$ & $0.070 \pm 0.026$ & $0.134 \pm 0.062$ & $0.142 \pm 0.043$ & $0.064 \pm 0.018$ & $0.108 \pm 0.020$ \\
\hline Galactose & & $0.108 \pm 0.018$ & $0.106 \pm 0.054$ & $0.099 \pm 0.026$ & $0.109 \pm 0.039$ & $0.094 \pm 0.018$ & $0.078 \pm 0.017$ & $0.077 \pm 0.014$ \\
\hline Glucose & Lactate & $0.626 \pm 0.105$ & $0.610 \pm 0.151$ & $0.936 \pm 0.132$ & $0.672 \pm 0.060$ & $0.648 \pm 0.133$ & $0.774 \pm 0.105$ & $0.661 \pm 0.094$ \\
\hline Galactose & & $0.626 \pm 0.105$ & $0.961 \pm 0.172$ & $0.758 \pm 0.100$ & $0.643 \pm 0.080$ & $0.542 \pm 0.107$ & $0.862 \pm 0.176$ & $0.605 \pm 0.081$ \\
\hline Glucose & ATP & $1.58 \pm 0.18$ & $1.92 \pm 0.25$ & $1.65 \pm 0.18$ & $1.73 \pm 0.20$ & $1.58 \pm 0.28$ & $1.68 \pm 0.24$ & $1.74 \pm 0.27$ \\
\hline Galactose & & $1.58 \pm 0.18$ & $1.36 \pm 0.15 \ddagger$ & $1.48 \pm 0.17$ & $1.54 \pm 0.15$ & $1.98 \pm 0.21$ & $1.49 \pm 0.24$ & $1.58 \pm 0.10$ \\
\hline
\end{tabular}

pups fed glucose or galactose at any individual time epoch. Glucose-6-phosphate levels were equivalent in both pup groups at each time period. Nonetheless, glucose-6-phosphate concentrations increased within $15 \mathrm{~min}$ and remained elevated at each time period except at $30 \mathrm{~min}$ for glucose-fed pups $(p<0.05)$. There were no significant changes for hepatic fructose-6-phosphate or fructose-1, 6 diphosphate levels across time or between groups (data not presented). Phosphoenolpyruvate concentrations in contrast increased compared with fasting levels after hexose feedings at 60 and $180 \mathrm{~min}$ for glucose $(p<0.05)$. Hepatic phosphoenolpyruvate was augmented in galactose-fed pups compared with glucose-fed pups $15 \mathrm{~min}$ after alimentation (Table 1). In contrast, pyruvate concentrations became lower than fasting levels at $15 \mathrm{~min}(p<0.02)$ and $180 \mathrm{~min}(p<0.05)$ after glucose feeding. There were no significant differences for hepatic lactate or citrate levels (data not presented) as a function of time or enteric hexose. Hepatic ATP concentrations were not altered by glucose alimentation. In contrast, hepatic ATP levels were lower at $15 \mathrm{~min}$ in pups fed galactose compared with glucose-fed pups. This difference did not persist after $15 \mathrm{~min}$ (Table 1).

There were no differences in the total or percent activity of glycogen synthase in either hexose-fed groups. Glycogen synthase was $38 \pm 3.1 \%$ in the active state during fasting and increased at $60 \mathrm{~min}$ to $72 \pm 5.5$ and $74 \pm 6.1 \%$ in glucose- and galactose-fed rats, respectively. Thereafter, glycogen synthase activation ranged between 72 and $88 \%$ without any time period demonstrating a difference of activation.

Glycogen phosphorylase was $100 \%$ in the active state during fasting and declined by 120 min to $81 \pm 8.8$ and $85 \pm 7.2 \%$ in glucose and galactose alimented pups. There were no differences between pup groups after this time with values ranging from 71 to $91 \%$ in the active state.

\section{DISCUSSION}

Glycogen metabolism. Galactose has been demonstrated to be preferentially incorporated into hepatic glycogen compared with glucose in perinatal and adult mammals $(2,3,6)$. Previous investigations by this laboratory in newborn dogs have demonstrated a reduced rate of systemic glucose appearance after enteric galactose administration compared with glucose-fed newborn dogs $(2,3)$. Given equivalent rates of absorption, these data suggested that there was augmented hepatic carbohydrate uptake when galactose was fed compared with glucose. Similar observations in the monkey fetus and adult rat have been noted by other investigators who demonstrated enhanced carbohydrate uptake with galactose as the hexose source in vitro $(5,6)$. The enhanced carbohydrate uptake in the presence of galactose could be due to augmented hepatic glycogen synthesis or carbohydrate oxidation (4). Previous studies in the newborn dog demonstrated enhanced incorporation of ${ }^{14} \mathrm{C}$ label from galactose into glycogen or actual net glycogen synthesis after galactose alimentation compared with that for glucose $(2,3)$. Nonetheless, the tissue in these studies was sampled 2-4 $\mathrm{h}$ after peak blood galactose concentrations, a time potentially far removed from maximal perturbations of tissue metabolites by galactose. Due to the prolonged time period between galactose feeding and tissue sampling, the immediate galactose-mediated effects on hepatic metabolism may be masked or hidden during the intervening unsampled time epochs.

In the present investigation we noted a dissociation between the effects of enteral galactose and glucose administration on hepatic glycogen synthesis in previously fasted newborn rats. This difference related to the rate of glycogen synthesis as galactose-fed rats demonstrated an immediate labeling of hepatic glycogen. This pattern could not be predicted from previous investigations of the effects of galactose on neonatal hepatic glycogen metabolism $(2,3,7)$. Within 15 min after feeding, the appearance of counts incorporated into hepatic glycogen increased rapidly and eventually achieved values far in excess of that after labeled glucose feedings at every time point. Label incorporation into hepatic glycogen is probably a more sensitive method of assessing flux of a precursor into glycogen. However, the appearance of label itself into glycogen does not demonstrate net glycogen synthesis. Nonetheless, when the actual quantity of assayable hepatic glycogen was determined galactose-alimented pups demonstrated a much earlier increase in net glycogen synthesis compared with basal fasting levels and with rat pups fed an equivalent amount of glucose. Net glycogen content remained elevated after galactose alimentation for 240 additional 
min. This rapid rise of net glycogen synthesis and appearance of label into glycogen was initiated when blood galactose concentrations and blood galactose radioactivity were elevated.

In contrast to the pattern of hepatic glycogen synthesis after galactose alimentation, net glycogen content did not increase to the same magnitude as rapidly after enteric glucose feeding. Indeed the peak for net glycogen synthesis after enteric glucose was delayed and appeared $240 \mathrm{~min}$ into the study. Furthermore, in contrast to the marked appearance of label incorporated into glycogen after galactose, ${ }^{14} \mathrm{C}$ appearing in glycogen after glucose feeding was attenuated. Radioactivity incorporated in glycogen after glucose feeding increased much more slowly than after galactose feeding and achieved peak values between $240-300$ min of the study. The pattern of net hepatic glycogen synthesis and label incorporation into glycogen after glucose feeding was out of phase with the peak glucose concentration that occurred at $60 \mathrm{~min}$ and the peak blood $\left[{ }^{14} \mathrm{C}\right]$ glucose radioactivity that also occurred at $60 \mathrm{~min}$. Indeed net glycogen synthesis in glucosefed rats occurred after blood glucose levels and blood $\left[{ }^{14} \mathrm{C}\right]$ glucose counts had already declined. These data suggest that galactose is more readily incorporated into neonatal hepatic glycogen both as a function of rate of synthesis and as a function of the magnitude of net glycogen synthesis. It is also suggestive that galactose may be more rapidly incorporated into glycogen by way of a preferential or more direct metabolic pathway.

Glucose incorporation into glycogen in adult mammals may be indirect after being converted to three carbon compounds, thus requiring recycling through gluconeogenesis for glycogen synthesis. The latter has been proposed as the primary mechanism of net glycogen synthesis after glucose alimentation in adult mammals (12-17). Previous work by other laboratories has demonstrated that glucose is not necessarily converted directly to glycogen (12-17). Instead, glucose diverts gluconeogenic precursors away from hepatic glucose production toward hepatic glycogen synthesis (17). Once converted to lactate, glucose carbons may then enrich the gluconeogenic precursor pool and function "indirectly" as a substrate for glycogen synthesis (12). Preliminary data from our laboratory support this "indirect" mechanism for neonatal murine hepatic glycogen synthesis from glucose (18). In the present study, the delayed time sequence for neonatal glycogen synthesis after glucose feeding also suggests that glucose is not directly incorporated into glycogen, as compared with galactose. Furthermore, the marked and consistent reduction of labeled carbon incorporation into glycogen after glucose alimentation suggests that this label was diluted in a different and larger pool than was the label for galactose. The greater radioactivity in glycogen after galactose alimentation suggests that the "galactose" precursor pool is more closely linked to glycogen synthesis than is the "glucose" pool. The delayed appearance of labeled glucose into glycogen compared with the more rapid incorporation of galactose suggests that further metabolism of glucose, possibly to three carbon gluconeogenic precursors may occur before incorporation of glucose-derived carbons into glycogen. Previous work in adult rats had demonstrated that lactate may be the predominant precursor for hepatic glycogen synthesis after oral glucose feeding (19). Indeed, the increase of lactate utilization could be accounted for by the increase of hepatic glycogen synthesis (19). However, galactose may be directly diverted toward glycogen synthesis after phosphorylation and conversion to UDP-glucose and subsequent entry into the glycogen synthesis pathway. Although glycogen synthase appeared to be activated and phosphorylase to be less active after hexose administration there were no differences at any time points between the pup groups. Furthermore, plasma insulin levels were lower in galactose-fed pups. These data suggest that the activities of the enzymes of glycogen synthesis and the presence of insulin may only be permissive for neonatal hepatic glycogen synthesis $(12,13,20)$. The more important factor in neonatal glycogen synthesis from galactose or glucose may be the availability or specificity of the substrate rather than the presence of elevated plasma insulin levels or additional activation or inactivation of glycogen synthase or phosphorylase $(2,6)$.

Hepatic intermediates. Previous studies in our laboratory have investigated the effects of enteric glucose and galactose alimentation on perturbations of hepatic glycolytic and Kreb cycle intermediates (7). Compared with completely fasted newborn dogs, both glucose and galactose resulted in increased concentrations of various hepatic metabolites. Nonetheless we did not observe differences between hepatic metabolites in newborn dogs after glucose or galactose alimentation (7). These alterations were noted $2-4 \mathrm{~h}$ after enteric alimentation and thus may not reflect the acute alterations of metabolites at the time of enteric hexose feeding.

Presently there is a paucity of information on the metabolic effects of oral carbohydrate substrates on neonatal hepatic metabolism. In the present neonatal investigation the most striking and perhaps clinically significant observation was the increase of hepatic galactose-1-phosphate concentrations after galactose alimentation. Similar observations of an increase of fructose-1phosphate levels were noted in adult rats fed fructose (21). Although the amount of galactose administered was a physiologic quantity, it may be concerning that galactose-1-phosphate levels increased after galactose alimentation and that this increment persisted after blood galactose levels had returned to normal. In contrast to newborn rats, hepatic galactose-1-phosphate levels were not elevated in newborn dogs $3-4 \mathrm{~h}$ after enteric galactose feeding. These data suggest that this response may be species related, but nonetheless, suggest that excessive galactose administration may result in accumulation of hepatic galactose-1phosphate and potentially produce adverse effects as noted in galactosemia (22).

Another concerning but more transient alteration is the reduction of hepatic ATP concentrations noted 15 minutes after galactose administration. Similar observations have been reported after fructose feeding in adult rats (23). In both studies glucose had no effect on ATP levels and the decline of ATP by both hexoses was transient. The reduction of ATP may result from rapid phosphorylation of galactose by galactokinase or a transient increase in adenine nucleotide deamination and probably has no clinical significance unless excessive quantities of galactose are administered $(23,24)$.

A decline of hepatic UDP-glucose occurred in both glucoseand galactose-fed pups at times when glycogen synthesis was present and when glycogen synthase was in the more active state. Similar observations of reduced hepatic UDP-glucose levels and enhanced glycogen synthase activity were noted in adult rats fed glucose during glycogen synthesis (19). With activation of glycogen synthase, substrates are "pulled" toward glycogen synthesis $(20,25)$. The observed alterations of UDP-glucose are compatible with this sequence.

Glucose-6-phosphate levels were elevated after both glucose and galactose feeding. The alteration of glucose-6-phosphate suggests that both carbohydrates enriched this intermediate pool $(19,21)$. Glucose would do this directly after phosphorylation by glucokinase (7). Galactose was readily converted to glucose during these current experiments as evident by the rapid appearance of ${ }^{14} \mathrm{C}$ label from galactose into circulating glucose. This pathway must pass through the hepatic glucose-6-phosphate pool and could explain the elevated hepatic glucose-6-phosphate concentrations in galactose-fed pups.

A consistent observation in both the present study and our previous work in newborn dogs after enteric hexose feeding has been an increase of hepatic phosphoenolpyruvate concentrations (7). Similar observations have been noted in vitro and in vivo with the addition of glucose to cell cultures or after glucose feeding to starved adult rats $(26,27)$. Phosphoenolpyruvate levels often increase during gluconeogenesis as pyruvate kinase activity is inhibited and pyruvate carboxylase activity is stimulated (2830). Although we would not expect active net gluconeogenesis, recycling of lactate (as a precursor) during glycogen synthesis 
could explain in part the observed increased phosphoenolpyruvate concentrations and the reduced hepatic pyruvate levels. These latter observations are compatible with the indirect and delayed appearance of glucose-derived carbons in hepatic glycogen after recycling of lactate in the gluconeogenic pathway.

\section{SUMMARY}

Sequential determinants of hepatic metabolism after glucose and galactose administration demonstrated marked differences. Glycogen synthesis was augmented earlier after galactose feeding whereas glucose-induced glycogen synthesis was delayed. Glucose carbon radioactivity incorporation into glycogen was attenuated relative to galactose, suggesting dilution of glucose in another intermediary pool, possibly the lactate pool. Persistent elevations of hepatic galactose-1-phosphate levels and a transient decline of ATP concentrations after galactose alimentation may not be of clinical significance but raises important concerns for the clinical use of galactose as the sole carbohydrate source for newborn mammals.

\section{REFERENCES}

1. Battaglia F, Sparks J 1983 Perinatal nutrition and metabolism. In: Boyd R Battaglia F (eds) Perinatal Medicine. Butterworths, London, pp 145-171

2. Kliegman R, Miettinen E, Kalhan S, Adam P 1981 The effect of enteric galactose on neonatal canine carbohydrate metabolism. Metabolism 30:1109-1118

3. Kliegman R, Miettinen E, Morton S 1983 Potential role of galactokinase in neonatal carbohydrate assimilation. Science 220:302-304

4. Segal S, Roth H, Bertoli D 1963 Galactose metabolism by rat liver tissue: influence of age. Science 142:1311-;1313

5. Sparks J, Lynch A, Glinsmann W 1976 Regulation of rat liver glycogen synthesis and activities of glycogen cycle enzymes by glucose and galactose. Metabolism 25:47-55

6. Sparks J, Lynch A, Chez R, Glinsmann W 1976 Glycogen regulation in isolated perfused near term monkey liver. Pediatr Res 10:51-56

7. Kliegman R, Miettinen E, Morton S 1983 Hepatic and cerebral energy metabolism after neonatal canine alimentation. Pediatr Res 17:285-291

8. Adam PAJ, Glazer G, Rogoff F 1975 Glucose production in the newborn dog. I. Effects of glucagon in vivo. Pediatr Res 9:816-820

9. Bergmeyer H 1974 Methods of Enzymatic Analysis, 2nd ed. Academic Press, New York

10. Vannucci RC, Duffy TE 1974 Influence of birth on carbohydrate and energy metabolism in rat brain. Am J Physiol 226:933-940
11. Schwartz AL, Rall TW 1973 Hormonal regulation of glycogen metabolism in neonatal rat liver. Biochem J 134:985-993

12. Boyd M, Albright E, Foster D, McGarry J 1981 In vitro reversal of the fasting state of liver metabolism in the rat. Reevaluation of the roles of insulin and glucose. J Clin Invest 68:142-152

13. Katz J, Golden S, Wals P 1979 Glycogen synthesis by rat hepatocytes. Biochem J 180:389-402

14. Katz J, McGarry J 1984 The glucose paradox. Is glucose a substrate for liver metabolism? J Clin Invest 74:1901-1909

15. Plas C, Chapeville F, Jacquot S 1973 Development of glycogen storage ability under cortisol control in primary cultures of fetal hepatocytes. Dev Biol 32:82-91

16. Radzivk J 1982 Sources of carbon in hepatic glycogen synthesis during absorption of an oral glucose load in humans. Fed Proc 41:110-116

17. Shikama H, Ui M 1978 Glucose load diverts hepatic gluconeogenic product from glucose to glycogen in vivo. Am J Physiol 235:E354-360

18. Kunst K, Kliegman R, Trindade C 1987 The glycogen synthesis glucose "paradox" in newborn rats. Pediatr Res 21:343A

19. Niewoehner C, Gilboe D, Nuttall F 1984 Metabolic effects of oral glucose in the liver of fasted rats. Am J Physiol 246:E89-E94

20. Newsholme E 1976 Carbohydrate metabolism in vivo: regulation of blood glucose level. Clin Endocrinol Metab 5:543-578

21. Niewoehner C, Gilboe D, Nuttall G, Nuttall F 1984 Metabolic effects of oral fructose in the liver of fasted rats. Am J Physiol 246:E505-E512

22. Gitzelmann $R$, Hansen $R 1980$ Galactose metabolism, hereditary defects and their clinical significance. In: Burman D, Holton J, Pennock C (eds) Inherited Disorders of Carbohydrate Metabolism. University Park Press, Baltimore, pp $61-101$

23. Woods H, Eggleston L, Krebs H 1970 The cause of hepatic accumulation of fructose-1-phosphate on fructose loading. Biochem J 119:501-510

24. Dormandy T, Leak D, Grant M 1959 Hypoglycemia induced by galactose. Lancet 2:269-272

25. Rollenston F 1972 A theoretical background to the use of measured concentrations of intermediates in the study of the control of intermediary metabolism. Curr Topics Cell Regul 5:47-75

26. Greenbaum AL, Gumaa KA, McLean P 1971 The distribution of hepatic metabolites and the control of the pathways of carbohydrate metabolism in animals of different dietary and hormonal status. Arch Biochem Biophys 143:617-625

27. Gumaa KA, McLean P 1969 The pentose phosphate pathway of glucose metabolism: enzyme profiles and transient and steady-state content of intermediates of alternative pathways of glucose metabolism in krebs ascites cells. Biochem J 115:1009-1014

28. Friedmann B, Goodman EH Jr, Saunders HL, Kostos V, Weinhouse S 1971 An estimation of pyruvate recycling during gluconeogenesis in the perfused rat liver. Arch Biochem Biophys 143:566-576

29. Ottaway JH, Mowbray J 1977 The role of compartmentation in the control of glycolysis. In: Horecker BL, Stadman ER (eds) Current Topics in Cell Regulation, Vol 12. Academic Press, New York, pp 108-209

30. Rognstad R: 1975 Cyclic AMP induced inhibition of pyruvate kinase flux in the intact liver cell. Biochem Biophys Res Commun 63:900-904 